\title{
Associated Risk Factors and Pulsed Field Gel Electrophoresis of Nasal Isolates of Staphylococcus aureus from Medical Students in a Tertiary Hospital in Lagos, Nigeria
}

\author{
Solayide A. Adesida ${ }^{1}$, Olusegun A. Abioye ${ }^{2}$, Babajide S. Bamiro ${ }^{3}$, Bartholomew I.C. Brai ${ }^{1}$, Stella I. Smith ${ }^{1}$, Kehinde O. Amisu ${ }^{3}$, \\ Deborah U. Ehichioya ${ }^{3}$, Folasade T. Ogunsola ${ }^{3}$ and Akitoye O. Coker ${ }^{3}$ \\ ${ }^{1}$ Molecular Biology and Biotechnology Division, Nigerian Institute of Medical Research; ${ }^{2}$ Dept. of Pharmaceutics and Pharmaceutical \\ Technology, Faculty of Pharmacy, University of Lagos; ${ }^{3}$ Department of Medical Microbiology and Parasitology, \\ College of Medicine, University of Lagos; Yaba, Lagos.
}

\begin{abstract}
Staphylococcus aureus infections are growing problems worldwide with important implications in hospitals. The organism is normally present in the nasal vestibule of about $35 \%$ of apparently healthy individuals and its carriage varies between different ethnic and age groups. Staphylococcal nasal carriage among health workers is particularly important to establish new clones and track origin of infections during outbreak situations. To determine the carriage rate and compare the pulsed field gel patterns of the strains, nasal swabs were collected from 185 medical students in a teaching hospital in Lagos, Nigeria. Isolates of $S$. aureus were tested for heamolysin production, methicillin sensitivity and Pulsed Field Gel Electrophoresis (PFGE) was performed. The results showed S.aureus nasal carrier rate of $14 \%$ with significant rate among males compared to females. All the isolates produced heamolysin. Antibiotic susceptibility pattern revealed that majority of the isolates was susceptible. Five strains (19\%) harboured resistant determinants to penicillin and tetracycline. None of the strains was resistant to methicillin. $44 \%$ of the isolates typed by PFGE had type B, the most predominant pulsotype. PFGE A clone exhibited a single resistance phenotype suggesting a strong clonal relationship that could punctual an outbreak in the hospital. The results speculate that nasal carriage among medical personnel could be a function of various risk factors. Personal hygiene and behaviour may however be the means to reducing colonization and spread of S.aureus in our hospitals.

Key-Words: Staphylococcus aureus, PFGE, nasal isolates, carriage rate.
\end{abstract}

Staphylococcus aureus are ubiquitous but the largest populations are found in the regions of the skin and mucous membranes surrounding openings in the body surface such as anterior nares, inguinal and perineal areas [1]. In apparently healthy adult population, $S$. aureus is present in the nasal vestibule of between $35 \%-50 \%$ of individuals at any one moment [2,3]. Inferences from longitudinal studies attempting to investigate frequency of factors that could influence nasal colonization in various populations and environmental conditions have suggested that nasal carriage may be higher in females than males and could be affected by hormonal status [4]. William et al. [5] was able to present data to show that carriage may also be influenced by occupation. They observed that $S$. aureus increased in the nose of a group of Malays and Indian footballers during aerobic training probably due to high oxygen demand.

Nasal carriage also varies among different ethnic and age groups [6,7]. There are considerable proofs that in healthcare settings, in-patients and hospital personnel are the main potential reservoirs of S.aureus. Basically, epidemiological pathways are from patients to healthcare worker to patients [8] and transmission to patients occurs during routine patient care [9]. For instance, a multivariate analysis of nasal carriage among otherwise healthy population particularly in hospital

Received on 15 August 2006; revised 27 January 2007.

Address for correspondence: Dr. Solayide Adesida. Molecular Biology and Biotechnology Division. Nigerian Institute of Medical Research, 6, Edmond Crescent, off Murtala Mohammed way, PMB 2013, Lagos. Email: slyesida@yahoo.com.

The Brazilian Journal of Infectious Diseases 2007;11(1):63-69. (C) 2007 by The Brazilian Journal of Infectious Diseases and Contexto Publishing. All rights reserved. environment showed that the frequency of carriage among paramedics of the Sedgwick County Emergence Medical Service in Wichita, Kansas was approximately $50 \%$ and the incidence remained high during the course of the study [10]. A similar study indicated that medical personnel were colonized with more antibiotic-resistant isolates than nonmedical personnel and the strain profiles revealed that they tended to be more clonal in origin, suggesting that exposure to hospital isolates alters colonization profile [9].

Regardless of these numerous studies that have investigated prevalence of nasal carriage of $S$. aureus and associated factors in developed countries, the subject has received limited attention in developing countries including Nigeria. While a study explained the role of S.aureus in wound infections and nasal carriage in staff of teaching hospital [11], another showed the resistance pattern of a set of nasal strains to commonly used antimicrobial agents [12], surprisingly; no studies that exploit molecular and statistical methods to explain the role of nasal carriage in transmission of S.aureus have been documented. In this present investigation, S.aureus colonization rates among medical students were assessed to indicate nasal carriage as a risk factor for successful transmission and development of staphylococcal infection in the country. To the best of our knowledge, this is the first documented report using Pulsed Field Gel Electrophoresis (PFGE) to establish the relation of nasal isolates in Nigeria.

\section{Materials and Methods}

Study Population

One hundred and eighty-five third-year medical students who were not aware of any ailment at the time of the sampling were screened for nasal colonization. Questionnaires 
concerning antibiotic use, habitual behaviour such as smoking and nose-picking and demographic details were administered. The study was carried out with the consent of each individual.

\section{Bacterial Isolates}

Participants were instructed on how to collect specimens by swabbing the surface of their nostrils with cotton swab (Evepon) as described previously [13]. The swabs were inoculated directly onto blood agar plate and then phenol red mannitol salt agar (Oxoid) at $37^{\circ} \mathrm{C}$ for 24 hours. Those isolates that were capable of fermenting mannitol were analysed further.

\section{Biochemical Identification}

Using standard microbiological protocol [14], colonies from pure culture were subjected to Gram staining reaction, catalase test, tube coagulase test and clumping factor/protein A kit (Slidex Staph Plus, Biomeriuex). Quality control was achieved with S.aureus ATCC 29213 strain for positive results and S.epidermidis ATCC 14990 strain for negative results.

\section{Haemolysis}

Isolates were tested for haemolysis after overnight incubation on plates of blood agar containing 5\% sheep blood. Cultures were scored as positive if a clear zone of $\beta$-haemolysis was observed on the blood agar.

\section{Antimicrobial Susceptibility Testing}

All isolates were screened for resistance to muprocin on Mueller-Hinton agar with a $5 \mu \mathrm{g}$ disk (Oxoid). An inhibition zone of $\leq 13 \mathrm{mM}$ in diameter was considered to reflect resistance [15]. Susceptibility to other antimicrobial agents was determined by disk diffusion method as recommended by the National Committee for Clinical Laboratory Standards [16] with the following: penicillin (10 Units), erythromycin $(15 \mu \mathrm{g})$, gentamycin $(10 \mu \mathrm{g})$,

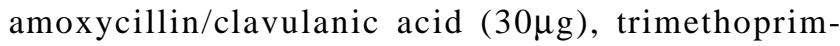
sulfamethoxazole (25 $\mu \mathrm{g})$, ceftazidime $(30 \mu \mathrm{g})$, cefuroxime

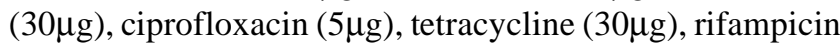

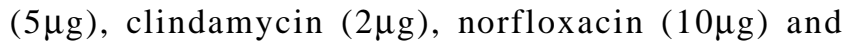
vancomycin $(30 \mu \mathrm{g})$. The susceptibility was performed on Mueller-Hinton agar (Oxoid) with S.aureus strain ATCC 25923 as a control. The plates were incubated at $35^{\circ} \mathrm{C}$.

\section{Methicillin Resistance Determination}

Methicillin resistance was determined with agar screen method with $4 \% \mathrm{NaCl}$ and oxacillin $(6 \mu \mathrm{g} / \mathrm{mL})$ NCCLS [16] and MRSA-latex agglutination test (Denka, Seiken Co., Ltd.). The MRSA screen test was performed according to the manufacturer's protocol (Denka, Seiken Co., Ltd.). The PBP2a was extracted by suspending a loopful of bacterial colonies around the oxacillin disc in four drops $(200 \mu \mathrm{L})$ of extraction reagent 1 in a $1.5 \mathrm{~mL}$ microphage tube. The suspension was mixed by vortexing and boiled for 3 minutes in a water bath. After cooling for 5 minutes at room temperature, one drop $(50 \mu \mathrm{L})$ of extraction reagent 2 was added, mixed and centrifuged at $1400 \mathrm{x}$ g for 5 minutes. Reagents 1 and 2 were supplied in the kit. The supernatant was then used for the latex agglutination test. Fifty microliters aliquot of the supernatant containing the PBP2a was added to each of two circles on disposable test cards labeled test and control. One drop of anti-PBP2a monoclonal antibody sensitized latex was added to the specimen on the test circle and one drop of the control negative latex was added to the control circle. The sample were mixed on a shaker for 3 minutes and assessed for agglutination virtually. MRSA ATCC 43300 strain was used as positive control while S.aureus ATTCC 29213 strain was for negative results.

\section{Pulsed Field Gel Electrophoresis (PFGE)}

PFGE procedures previously described for S.aureus [17] was performed. Three discrete colonies of $S$. aureus cells from an overnight culture were suspended in $100 \mu \mathrm{L}$ of EET buffer (100mM EDTA.10mM EGTA. 10mMTris-HCl)-pH 8). The bacterial suspension was added to an equal volume of lowmelting point 1\% InCert agarose (FMC Bioproducts, Rockland, Maine), mixed thoroughly before casting into plugs. The gel plugs were incubated for four hours in lysostaphin solution (Sigma Chemicals Co.), de-proteinised by incubating overnight at $37^{\circ} \mathrm{C}$ and about $1 / 4$ was equilibrated in smaI digestion buffer (Boehringer-Mannheim, Mannheim, Germany). After addition of 20 units smaI, the blocks were incubated overnight at room temperature. The plugs were embedded in a $1 \%$ agarose gel and subjected to contour clamped homogeneous electric field gel electrophoresis in a BioRad CHEF Mapper. The total run time amounted to $23 \mathrm{hrs}$, with a first block during which the switch time increased from 5 to 15 s over a period of $10 \mathrm{hr}$ and a second block of $13 \mathrm{hr}$ with a switch time increase from 15 to $60 \mathrm{~s}$. The incubation temperature was $14^{\circ} \mathrm{C}$, the voltage $6 \mathrm{~V} /$ $\mathrm{cm}$, linear ramping was applied and the included angle was $120^{\circ}$. Gels were stained in ethidium bromide solutions and photographed under ultraviolet light using whole BioImage analyzer. Banding patterns were inspected and compared visually. Strains identical in size and number of bands were considered genetically indistinguishable and assigned to the same type; strains with banding patterns that differed by only three or fewer bands were considered closely related and described as subtypes of a given clonal type and strains with banding patterns that differed by four or more bands were considered different and assigned to separate types [18].

\section{Statistical Analysis}

The responses to the questionnaire were manually coded for and the data was analysed using Epi-Info computer software version 6.0 (from the Centers for Disease Control and Prevention). A p value of $<0.05$ (5\%) was taken as statistically significant while $\mathrm{p}>0.05$ was taken as nonsignificant. 


\section{Results}

One hundred and eighty-five (185) medical students comprising of ninety-one female and ninety-one male were enrolled in the study. Three students did not indicate their gender; therefore the overall statistical analyses were based on one hundred and eighty-two (182) students. Questionnaire data for the 182 students indicated that all were Nigerians. The demographic profile and other parameters of the study participants are summarized in Table 1. Most of the subjects were from Yoruba ethnic group of the southwestern part of the country (62.1\%), followed by Igbo (Eastern part) (25.8\%) and Edo (2.7\%) and Urhobo (2.2\%) (Southsouth). The male to female sex ratio was 1:1. Only twenty-six (14\%) of the subjects analyzed carried S.aureus in their anterior nares. Subjects whose nasal specimens did not test positive for S.aureus were classified as S.aureus negative.

Differential function analysis was performed statistically to evaluate the contribution of the following factors: tribe, age, gender, health status and use of antibiotic, personal hygiene and habits to S.aureus colonisation in the twentysix carriers. There were no significant differences in carriage rate and any previously or currently used antibiotic (s). Other possible risks factors such as gender, smoking, past or present alcohol use, hand-washing habits and method of cleaning the nostrils were also observed in frequencies not significant statistically. However, the outcome of positive S. aureus cultures was significantly affected by age (p-value: $0.004096 ; \chi^{2}: 37.82$; df: 18) (Figure 1 ) and highest prevalence was established among the subjects in the age group 21-25. Tribe also has important implication on carriage (p-value: 0.01049586; $\chi^{2}: 26.07 ; \mathrm{df}: 12$ ) (Figure 2) and males were more affected than the females

All the twenty-six S.aureus strains analyzed were $\beta$ heamolytic and fully susceptible to muprocin, cefuroxime, amoxycillin/clavulanic acid, ciprofloxacin, vancomycin, rifampicin and norfloxacin but resistant to penicillin. Five strains (19\%) harboured resistant determinants to penicillin and tetracycline. Resistance to a combination of penicillin and ceftazidime was observed in nine strains (35\%). While four $(15 \%)$ of the strains showed a combined resistance to penicillin, tetracycline and trimethoprim-sulfamethoxazole, three (12\%) were resistant to penicillin and trimethoprimsulfamethoxazole. Only a single strain showed four determinants (Figure 3). None of the isolates were resistant to methicillin. The sizes of inhibition zone in the oxacillin disk diffusion test ranged from $14-25 \mathrm{mM}$ and there was no inhibition of growth in the agar screen test.

Sixteen of the strains were randomly selected and analyzed for their genetic relatedness. Pulsed Field Gel Electrophoresis revealed carriers of an identical strain in seven of the subjects. $43.75 \%$ of the nasal isolates (7 strains) had type $\mathrm{B}$, the most predominant pulsotype. The second most prevalent clone was type A with a single subtype. Five of the seven PFGE types were unique as a single type (C, D,
E, F, and G). A total of seven different PFGE profiles were detected (Figure 4). PFGE type A was highly associated with resistance to penicillin. We however did not find any correlation between PFGE genotype, age and sex (Table 2) of the students.

\section{Discussion}

The anterior nares have been described as primary ecologic niche for Staphylococcus aureus and nasal colonization by this opportunistic pathogen is known to increase the risk of development of S.aureus infections. This present study identifies the importance of health-workers as agents of transmission in the hospital and establishes the genetic relationship among the strains in Nigeria. The population investigates is unique in that medical students are believed to have brief but often uncontrolled exposures to patients. Sometimes, they may be responsible for transporting patients within the hospitals facilities. The nasal carrier rate of S.aureus in this present study was $14 \%$. This carriage rate correlates well with the results of Chigbu and Ezeronye [12] and the ranges described by Riewerts et al. [19] but lower than the figures obtained in another Nigerian study [6]. This variation may be attributed to the population studied, the sampling and culture techniques. Lamikanra et al. [6] observed that carriage rate of individuals from 9-32 years decrease progressively as the age increases. The present investigation has mean age of 24 .

Contrary to previous observation [20], gender seems to have no effect on the carriage rate and neither did it appear that certain factors such as "nose picking" are important predisposing factors to colonization in the populations analyzed in this study. Cespedes et al. [9] however found that hospital staff have higher incidence of hand and nasal colonization with S.aureus and could serve a vectors of S.aureus transmission to hospitalized patients. The results presented here may be attributed to the fact that the population comprises of medical student who are aware of the importance of handwashing in hospital environment. As such, the bacteria carried on the hands, which could have been transferred to the nostrils during "nose-picking" process, would have been taken care of during handwashing.

Although, the strains analyzed in this study were not representative samples of the various tribes presented because of the geographical location of the study centre, nevertheless, the findings indicated that tribe could be a significant predisposing factor to S.aureus colonization. This may be a function of metabolic activity and skin type or colour of individuals. Also in study of a group of young Malaysians and Indians footballers, William and co-workers [5] explained that sweat produced from the skin contains lysozyme, a natural body defense substance responsible for protecting the body from infection and has the ability to lyse and inhibit bacteria. Studies are therefore needed to confirm that nasal carriage is occurring more frequently in certain ethnic group than others. 
Table 1. Summary of some of the demographic Profiles of subjects screened for Staphylococcus aureus nasal colonization

\begin{tabular}{lcc}
\hline Variable & Frequency (\%) & Percentage \\
\hline Age [year (mean: 23.9)] & 48 & 26 \\
$<21$ & 103 & 57 \\
$21-25$ & 6 & 3 \\
$26-30$ & 25 & 14 \\
$>31$ & & \\
Sex & 91 & 50 \\
Male & 91 & 50 \\
Female & & \\
Tribe & 113 & 62.1 \\
Yoruba (Southwest) & 47 & 25.8 \\
Igbo (SouthEast) & 12 & 7.0 \\
Edo (Southsouth) & 2 & 1.1 \\
Ibibio (Southsouth) & 2 & 1.1 \\
Tiv (Northcentral) & 6 & 3.0 \\
Others & & \\
Subjects who & 1 & 0.5 \\
Had any underlying diseases & 2 & 1.1 \\
Were recently hospitalized & 4 & 2.2 \\
Were smokers & 9 & 4.9 \\
Were alcohol users & 97 & 53.3 \\
Were cleaning nostrils regularly & 63 & 34.6 \\
Were cleaning nostrils with fingers & 107 & 58.8 \\
Were washing hands regularly & 21 & 11.5 \\
Frequently use antibiotics & 66 & 36.3 \\
Were taking antibiotics on prescription & 48 & 26.4 \\
Were taking antibiotics on self-medication & 7 & 4.0 \\
Currently taking antibiotics or within the last two weeks & 32 & 18.0 \\
Were frequent users of common ß-lactams & & \\
\hline
\end{tabular}

Figure 1. Relationship between age and Staphylococcus aureus nasal carriage.

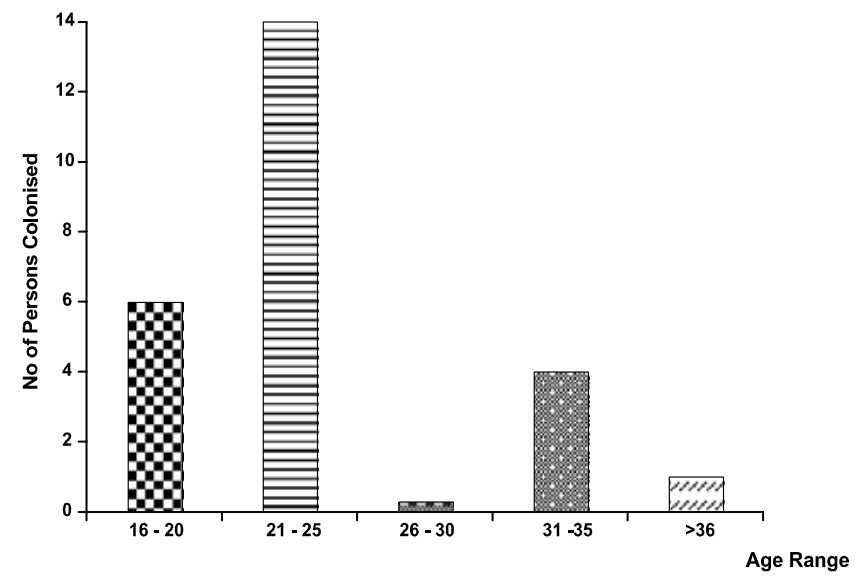

Figure 2. Frequency of Carriage among various tribes.

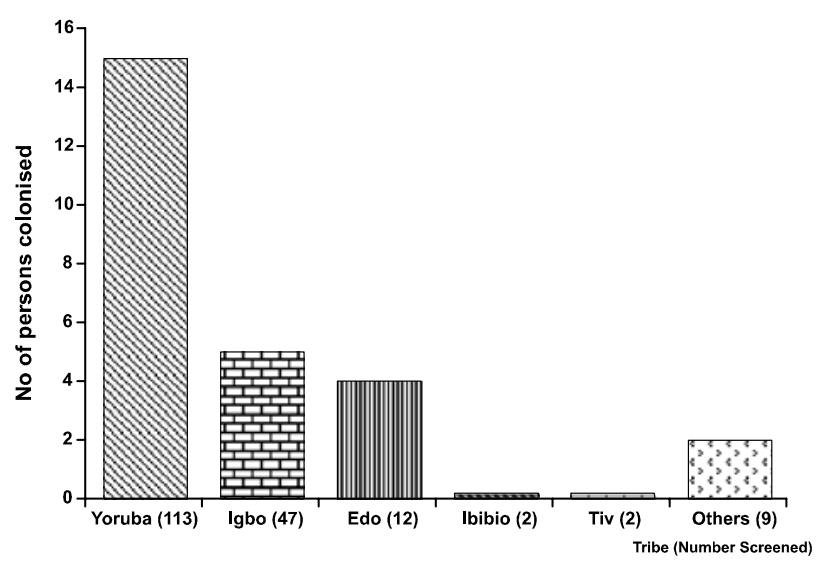


Figure 3. Resistance phenotypes of nasal isolates.

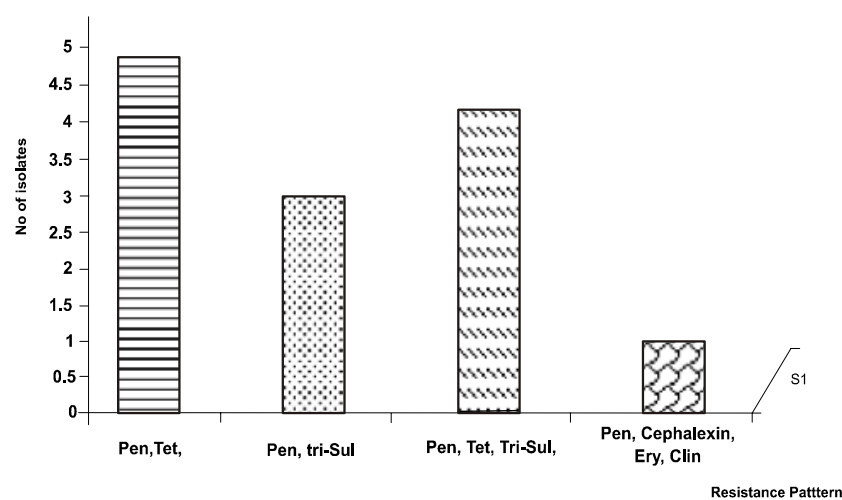

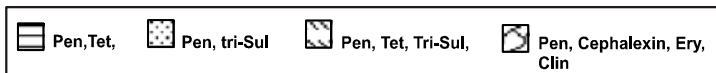

Pen: penicillin; Tet: tetracycline; Ceft: ceftazidime; Tri-Sul: trimethoprim-sulfamethoxazole; Ery: erythromycin; Clin: clindamycin.
Table 2. Characteristics of sixteen S.aureus strains

\begin{tabular}{lllll}
\hline $\begin{array}{l}\text { Isolate } \\
\text { code }\end{array}$ & Age & Sex & $\begin{array}{l}\text { PFGE } \\
\text { Pattern }\end{array}$ & $\begin{array}{l}\text { Antibiotic } \\
\text { resistant pattern }\end{array}$ \\
\hline LM69 & 21 & Male & A & Pen \\
LM79 & 22 & Female & A & Pen \\
LM 85 & 33 & Male & A & Pen \\
LM 88 & 21 & Male & B & Pen \\
LM90 & 34 & Male & C & Pen \\
LM91 & 25 & Female & B1 & Pen, Tet, Tri/sulf \\
LM105 & 20 & Female & B2 & Pen, Tri/sulf \\
LM119 & 20 & Female & D & Pen \\
LM133 & 21 & Male & A1 & Pen \\
LM138 & 20 & Female & B & Pen, Tet \\
LM146 & 21 & Female & B3 & Pen, Tet \\
LM148 & 20 & Female & E & Pen \\
LM154 & 21 & Male & F & Pen \\
LM158 & 20 & Female & B4 & Pen, Tri/sulf \\
LM160 & 21 & Female & G & Pen, Tri/sul \\
LM185 & 21 & Female & B & Pen, Tet, Tri/sulf \\
\hline
\end{tabular}

Pen: penicillin, Tet: tetracycline, Tri/sulf: trimethoprimsulfamethoxazole.

Figure 4. Fingerprints of sixteen strains obtained smaI-PFGE.

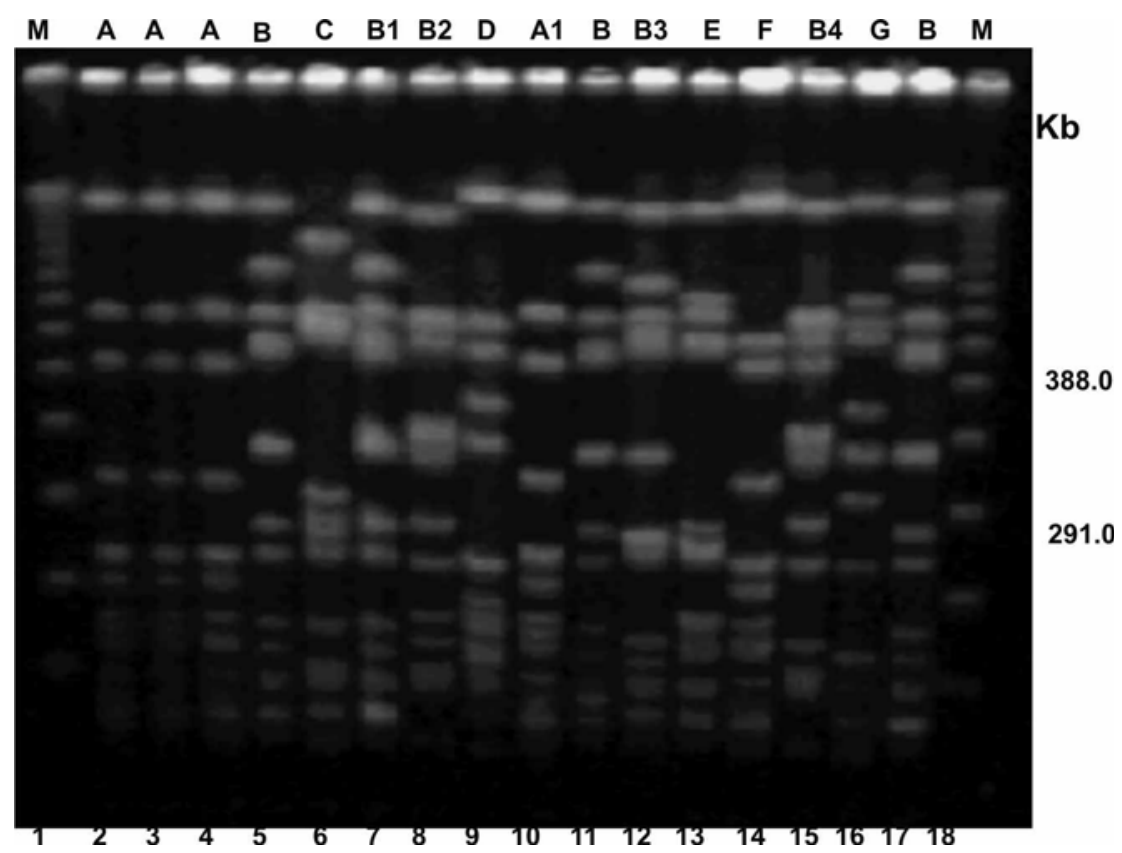

Lane1 and Lane18: molecular weight marker (Lambda Ladder with size range of 50-1000kilobase).

$\begin{array}{lll}\text { Lane 2:LM69 } & \text { Lane 6: LM90 } & \text { Lane 7: LM91 } \\ \text { Lane 3: LM79 } & \text { Lane 9: LM119 } & \text { Lane 10: LM133 } \\ \text { Lane 4:LM85 } & \text { Lane 12: LM146 } & \text { Lane13: LM148 } \\ \text { Lane 5:LM88 } & \text { Lane 15: LM158 } & \text { Lane 16:LM160 }\end{array}$

Lane 8: LM105

Lane 11: LM138

Lane14: LM154

Lane 17: LM185 
The identity of the S.aureus isolates from each individual was first established phenotypically by heamolysin production and antibiotic susceptibility pattern. Most of the isolates appeared to be $\beta$-haemolytic and the antimicrobial susceptibility testing revealed a preponderance of susceptible strains. The strains were primarily susceptible to the antimicrobial agents tested except penicillin, tetracycline, ceftazidime and trimethoprim-sulfamethozle. This finding is consistent with antibiotic susceptibility patterns reported in a previous study [12] and contrast sharply with multidrug patterns often observed in industrialized countries [21,22]. The absence of mecA gene is consistent with previous report on clinical isolates [23] and shows that methicillin resistant S.aureus are indeed of low prevalence in Nigeria.

Macrorestriction and Pulsed field gel electrophoresis is well known in being superior in typeability and reproducibility to any typing methods and allows an excellent discrimination between strains [24]. Sixteen of the twentysix strains were investigated genotypically using PFGE. The results generated clearly support other studies [10] and show that hospital personnel particularly medics appear to be a major reservoir for staphylococcal infections and indeed important vector for transmission of S.aureus within the hospital setting. Among the sixteen strains, a major clone (PFGE type B) containing about $44 \%$ of the isolates was identified. This suggests the possible transmission of a single strain among the group and also strengthens the hypothesis that S.aureus is clonal in nature. It might be further interesting to know whether nasal strains from hospital personnel would be genetically identical to clinical strains circulating within the same hospital.

Nevertheless, the resistance profile was correlated with the PFGE types. Although it has been shown that strains with similar genetic make-up may necessarily not have the same antibiogram [25], in this study, we found that antibiogram seems a useful adjunct to existing epidemiologic typing methods probably because of the apparently low resistance observed among the S.aureus population. Looking at the sixteen strains analyzed by PFGE, the members of PFGE type A exhibited a common resistance phenotype (Pen). Since this emphasizes their close relatedness or possible common origin, it could be inferred that a clone with high dissemination potential, which might be disturbing in an outbreak situation, is present in the hospital. Consequently, it would be necessary to introduce strict surveillance measures in the hospital.

In conclusion, the results of the observations described herein show clearly that nasal colonization among medical personnel is a function of various risk factors. Personal hygiene and behaviour may however be the key to reducing colonization and spread of S.aureus. Handwashing seems to reduce colonization rate and therefore, it would be proposed that infection control intervention through handwashing be strictly adhered to.

\section{References}

1. Saxena S., Singh K., Talwar V. Methicillin-Resistant Staphylococcus aureus Prevalence in Community in the East Delhi Area. Jpn J Infect Dis 2003;56:54-6.

2. Uemura E., Kakinohana S., Higa N., et al. Comparative Characterization of Staphylococcus aureus Isolates from Throats and Noses of Healthy Volunteers. Jpn J Infect Dis 2004;57:21-4.

3. Nashev D., Toshkova K., Isrina S., et al. Distribution of virulence genes of Staphylococcus aureus isolated from stable nasal carriers. FEMS Microbiol Lett 2004;233:45-2.

4. Winkler J., Block C., Leibovici L., et al. Nasal carriage of Staphylococcus aureus: correlation with hormonal status in women. J Infect Dis 1990;162:1400-2.

5. William J. L., Radu S., Aziz S. A., et al. Prevalence of Staphylococcus aureus carriage by young Malaysian footballers during indoor training. Br J Sports Med 2004;38:12-4.

6. Lamikanra A., Paul B.D., Akinwole B.O., Paul M.O. Nasal Carriage of Staphylococcus aureus in a population of healthy Nigerian students. J Med Microbiol 1985;19:211-6.

7. Peacock S.J., Justice A., Griffiths D., et al. Determinants of acquisition and carriage of Staphylococcus aureus in infancy. J Clin Microbiol 2003;41:5718-25.

8. Kluytmans J., VanBelkum A., Verbrugh H. Nasal carriage of Staphylococcus aureus: Epidemiology, Underlying Mechanisms and Associated Risks. Clin Microbiol Rev 1997;10:505-20.

9. Cespedes C., Miller M., Quagliarello B., et al. Differences between Staphylococcus aureus Isolates from Nonmedical Hospital Personnel. J Clin Microbiol 2002;40:2594-7.

10. Elliot M.J., Kellum M.T., Pettriess R.L. Nasal carriage of methicillin-susceptible and methicillin-resistant Staphylococcus aureus among paramedics in the Sedgwick County Emergency Medical Service in Wichita, Kansas. Infect Control Hosp Epidemiol 2002;23:60-1.

11. Wemambu S.N.C. Wound Infections and Nasal colonization of Staff with Staphylococcus aureus in Benin, Nigeria. J Hosp Infect $1981 ; 2$ :259-60.

12. Chigbu C.O., Ezeronye O.U. Antibiotic resistant Staphylococcus aureus in Abia State of Nigeria. Afr J Biotech 2003;2:374-8.

13. Ahmed A.O., VanBelkum A., Fahal H.A., et al. Nasal carriage of Staphylococcus aureus and Epidemiology of surgical site infections in Sudanese University Hospital. J Clin Microbiol 1998;36:3614-8.

14. Cowan S.T., Steel K.J. Cowan and Steel's Manual for Identification of Medical Bacteria. Cambridge University Press, Great Britain, 1993.

15. Chaves F., Garci-Martinez J., DeMiguel S., Otero R. Molecular Characterisation of resistance to mupirocin methicillinsusceptible and resistant isolates of Staphylococcus aureus from nasal samples. J Clin Microbiol 2004;42:822-4.

16. National Committee for Clinical Laboratory Standards. Performance Standards for antimicrobial disk diffusion susceptibility tests, $7^{\text {th }}$ Ed. Approved Standard National Committee for Clinical Laboratory Standards, Wayne Pa., 2000.

17. Cookson B.D., Aparicio P., Deplano A., et al. Inter-centre comparison of pulsed-field electrophoresis for the typing of methicillin-resistant Staphylococcus aureus. Med Microbiol 1996; 44:179-84.

18. Tenover F.C., Arbeit R.D., Goering R.V., et al. Interpreting chromosomal DNA restriction patterns produced by pulsedfield gel electrophoresis: criteria for bacterial strain typing. J Clin Microbiol 1995;33:2233-9.

19. Riewerts E.N.H., Espersen F., Thamdrup R.V., Jensen K. Carriage of Staphylococcus aureus among 104 healthy persons during a 19-month period. Epidemiol Infect 1995;115:51-60.

20. Eriksen N.H., Esperssen F., Rosdahl V.T., Jensen K. Carriage of Staphylococcus aureus among 104 healthy persons during a 19-month period. Epidemiol Infect 1995;115:51-60. 
21. Safdar N., Narans N., Gorddon B., Maki D. G. Comparison of culture screening methods for detection of nasal carriage of methicillin-Resistant Staphylococcus aureus: a prospective study comparing 32 methods. J Clin Microbiol 2003;41:3163-6.

22. Campbell K.M., Vaughn F.A., Kevin L., et al. Risk factors for community-associated methicillin-resistant Staphylococcus aureus infections in an outbreak of disease among military trainees in San Diego, California, in 2002. J Clin Microbiol 2004;42:4050-3.
23. Adesida S., Boelens H., Bamiro B. et al. Major epidemic clones of Staphylococcus aureus in Nigeria. Microb Drug Resist 2005;11:115-21.

24. Tenover F.C., Arbeit R.D., Goering R.V. How to select and interpret molecular typing methods for epidemiological studies of bacterial infections: a review for healthcare epidemiologists. Infect Control Hosp Epidemiol 1997;18:426-39.

25. Tenover F.C., Arbeit R., Archer G., et al. Comparison of traditional and molecular methods of typing isolates of Staphylococcus aureus. J Clin Microbiol 1994;32:407-15. 